\title{
On the number of steady states of a climate energy balance model
}

Cite as: AIP Conference Proceedings 2116, 030025 (2019); https://doi.org/10.1063/1.5114009 Published Online: 24 July 2019

\section{A. Hidalgo, and L. Tello}

\section{ARTICLES YOU MAY BE INTERESTED IN}

Numerical simulation of a porous-medium type two-dimensional atherosclerosis model AIP Conference Proceedings 2116, 030033 (2019); https://doi.org/10.1063/1.5114017

Multiresolution scheme for two-phase volcanic flows

AIP Conference Proceedings 2116, 030024 (2019); https://doi.org/10.1063/1.5114008

Preface: International Conference on Numerical Analysis and Applied Mathematics (ICNAAM-2018)

AIP Conference Proceedings 2116, 010001 (2019); https://doi.org/10.1063/1.5113978

\section{Challenge us.}

What are your needs for periodic signal detection?

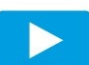

Watch

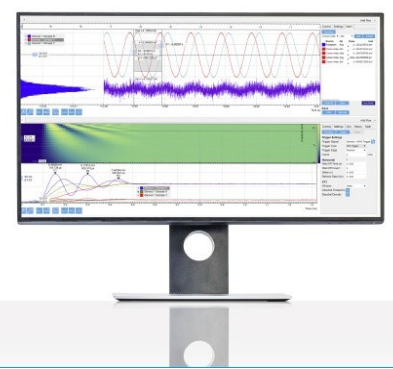




\title{
On the Number of Steady States of a Climate Energy Balance Model
}

\author{
A. Hidalgo ${ }^{1,3, b)}$ and L. Tello ${ }^{2,3, a)}$ \\ ${ }^{1}$ Dept. Ingeniería Geológica y Minera. E.T.S.l. Minas y Energía, Universidad Politécnica de Madrid, Rios Rosas 21, \\ 28003 Madrid, Spain. \\ ${ }^{2}$ Dept. Matemática Aplicada. ETS Arquitectura. Universidad Politécnica de Madrid. Av. Juan de Herrera, 28040 \\ Madrid (Spain). \\ ${ }^{3}$ Center for Computational Simulation, Universidad Politécnica de Madrid, Spain. \\ a)Corresponding author: 1.tello@upm.es \\ b) arturo.hidalgo@upm.es
}

\begin{abstract}
We are concerned with a global climate model with deep ocean effect. The model includes a dynamic and diffusive boundary condition arising from an energy balance on the Earth surface. The scales of time and space are relatively large. One of the characteristics of climate energy balance models is the sensitivity to the variations of a Solar parameter $Q$. Analytically, it was proved that there exists a range of $Q$ with at least three stationary solutions. In this work we analyze numerically the existence of more than three stationary solutions according to that positive parameter $\mathrm{Q}$.
\end{abstract}

\section{INTRODUCTION}

The model consists of an energy balance model of one layer coupled with a deep ocean model. The motivation of the study of this type of climate model is to show the relationship between the rapid climatic change in Glacial - Holocene transition and the past changes in deep water formation. One of the main difficulties is the mathematical treatment of the dynamic and diffusive boundary condition as well as the term of coalbedo (Budyko type). This kind of models are very sensitive to small fluctuations of the parameters. In particular, the number of stationary solutions depends on the value of the Solar constant.

The model is based on one proposed by Watts and Morantine [1] and completed with the nonlinear diffusion at the boundary proposed by P.H. Stone ([2]) for climate energy balance models. Concerning climate energy balance models there are some formulations on manifolds (see e.g. [3], [4] and references therein where the surface temperature is defined on a manifold simulating the Earth surface) and others which assume constant temperature over each parallel. The pioneering climate energy balance models were introduced by Budyko [5] and Sellers [6] in 1969.

In this work we consider the averaged temperature over each parallel as the unknown. So, it is possible to consider as one of the space variable the sine of the latitude $\alpha$, that is, $x=\sin \alpha$. Then, Laplacian of $u$ is expressed by using the spherical coordinates, $\Delta u=\left(\left(1-x^{2}\right) u_{x}\right)_{x}$ where the subscript $x$ denotes the derivative with respect to $x$. The p-Laplacian operator defined by $\Delta_{p} u:=\operatorname{div}\left(|\nabla u|^{p-2} \nabla u\right), p \geq 2$, includes the linear diffusion, $\mathrm{p}=2$, as a particular case and for $p=3$ the case proposed by P.H. Stone ([2]). Considering the simplification $x=\sin \alpha$ we have $\left(\left(1-x^{2}\right)^{\frac{p}{2}}\left|u_{x}\right|^{p-2} u_{x}\right)_{x}$.

Previous results prove multiplicity of stationary solutions for some values of a Solar parameter. In this work we obtain new stationary solutions for the coupled model. In the next section we describe the mathematical model.

\section{THE MODEL}

The model represents the evolution of the temperature in a 'global' ocean $\Omega$ with depth $H$. The unknowns are the ocean temperature, $U$, and the surface temperature, $u$. The space variables $(x, z), x=\sin \alpha$ (where $\alpha$ is the latitude)

International Conference of Numerical Analysis and Applied Mathematics (ICNAAM 2018)

AIP Conf. Proc. 2116, 030025-1-030025-3; https://doi.org/10.1063/1.5114009

Published by AIP Publishing. 978-0-7354-1854-7/\$30.00 
and $-z$ is the depth. The space domain is $\Omega=(-1,1) \times(-H, 0)$ and the boundary, $\Gamma_{H} \cup \Gamma_{0} \cup \Gamma_{1} \cup \Gamma_{-1}$, where $\Gamma_{H}=\{(x, z) \in \bar{\Omega}: z=-H\}, \Gamma_{0}=\{(x, z) \in \bar{\Omega}: z=0\}, \Gamma_{1}=\{(x, z) \in \bar{\Omega}: x=1\}, \Gamma_{-1}=\{(x, z) \in \bar{\Omega}: x=-1\}$.

We consider the problem

$$
(1) \begin{cases}\frac{\partial U}{\partial t}-\frac{K_{H}}{R^{2}} \frac{\partial}{\partial x}\left(\left(1-x^{2}\right) \frac{\partial U}{\partial x}\right)-K_{V} \frac{\partial^{2} U}{\partial z^{2}}+w \frac{\partial U}{\partial z}=0 & \Omega \times(0, T), \\ \frac{\partial u}{\partial t}-\frac{D K_{H_{0}}}{R^{2}} \frac{\partial}{\partial x}\left(\left(1-x^{2}\right)^{\frac{p}{2}}\left|\frac{\partial u}{\partial x}\right|^{p-2} \frac{\partial u}{\partial x}\right)+\mathcal{G}(u)+K_{V} \frac{\partial U}{\partial n}+w x \frac{\partial u}{\partial x} \in Q S(x) \beta(u)+f(x) & \Gamma_{0} \times(0, T) \\ U_{\Gamma_{0}}=u & \Gamma_{0} \times(0, T) \\ w x \frac{\partial U}{\partial x}+K_{V} \frac{\partial U}{\partial z}=0 & \Gamma_{H} \times(0, T) \\ \left(1-x^{2}\right)^{\frac{p}{2}}\left|\frac{\partial U}{\partial x}\right|^{p-2} \frac{\partial U}{\partial x}=0 & \left(\Gamma_{1} \cup \Gamma_{-1}\right) \times(0, T) \\ U(x, z, 0)=U_{0}(x, z) & \Omega \\ u(x, 0)=u_{0}(x) & \Gamma_{0} .\end{cases}
$$

The first equation is the governing equation of the deep ocean (DOM), where $U(x, z, t)$ represents the ocean temperature, $K_{V}$ the vertical thermal diffusivity, $K_{H}$, the horizontal thermal diffusivity in the ocean, $w$ the vertical velocity and $R$ is the Earth radius. The second equation is the dynamic and diffusive boundary condition at $\Gamma_{0}$ which arises from an energy balance on the surface (EBM). In the balance, $\mathcal{G}(U)$ represents the emitted energy (by cooling), $D$ is the depth of the mixed layer, $K_{H_{0}}$ is the horizontal thermal diffusivity in the mixed layer. The absorbed energy is given by the term $Q S(x) \beta(u)$ where $S(x)$ is the insolation, $\beta(u)$ is the planetary coalbedo and $Q$ is a Solar constant.

We assume

$\left(\mathrm{H}_{\beta}\right) \beta$ is a bounded maximal monotone graph of $\boldsymbol{R}^{2}$ with $D(\beta)=\boldsymbol{R}$ and there exist two real numbers $0<m<M$ and $\epsilon>0$ such that $\beta(r)=\{m\}$ for any $r \in(-\infty,-10-\epsilon)$ and $\beta(r)=\{M\}$ for any $r \in(-10+\epsilon,+\infty)$.

$\left(\mathrm{H}_{S}\right) S:(-1,1) \rightarrow R, \quad S \in L^{\infty}\left(\Gamma_{0}\right), \quad S_{1} \geq S(x) \geq S_{0}$ for some $S_{1}>S_{0}>0$.

$\left(\mathrm{H}_{\mathcal{G}}\right) \mathcal{G}: R \rightarrow R$ is a continuous strictly increasing function such that $\mathcal{G}(0)=0$. and $\lim _{|s| \rightarrow \infty}|\mathcal{G}(s)|=+\infty$.

$\left(\mathrm{H}_{f}\right) f \in L^{\infty}\left(\Gamma_{0}\right)$ and there exists $C_{f}>0$ such that $-\|f\|_{\infty} \leq f(x) \leq-C_{f}$ a.e. $x \in \Gamma_{0}$.

$\left(\mathrm{H}_{C_{f}}\right) \mathcal{G}(-10-\epsilon)+C_{f}>0$ and $\frac{\mathcal{G}(-10+\epsilon)+\|f\|_{\infty}}{\mathcal{G}(-10-\epsilon)+C_{f}} \leq \frac{S_{0} M}{S_{1} m}$.

$\left(\mathrm{H}_{w}\right) w \in C^{1}(\bar{\Omega})$.

The framework of problem (1) is constructed from the following functional spaces

$$
\begin{gathered}
V(\Omega)=\left\{U \in L^{2}(\Omega):\left(1-x^{2}\right)^{\frac{1}{2}} \frac{\partial U}{\partial x} \in L^{2}(\Omega), \frac{\partial U}{\partial z} \in L^{2}(\Omega)\right\}, \\
V p\left(\Gamma_{0}\right)=\left\{u \in L^{2}\left(\Gamma_{0}\right):\left(1-x^{2}\right)^{\frac{1}{2}} \frac{\partial u}{\partial x} \in L^{p}\left(\Gamma_{0}\right)\right\} .
\end{gathered}
$$

\section{MULTIPLE STATIONARY SOLUTIONS}

We are interested in the stationary solutions of the model described in previous section. In the work [7] it is proved that if $Q$ is small enough or big enough then there exists only one stationary solution for problem (1). The proof uses a comparison principle for an auxiliary problem. Moreover, an interval of $Q$ with at least three stationary solutions was found in the mentioned work. More precisely, there exist four positive constants, $0<Q_{1}<Q_{2}<Q_{3}<Q_{4}$, such that if $0<Q<Q_{1}$ or $Q_{4}<Q$, then (1) has a unique stationary solution. If $Q_{2}<Q<Q_{3}$, then (1) has at least three stationary solutions.

The purpose of the present work is to find more than three stationary solutions by using the approximation by the evolution solution by choosing suitable initial datum. To do that we use some qualitative properties of the $\omega$-limit set of the evolution solutions. 
We can focus our attention on the case $Q_{2}<Q<Q_{3}$. We analyze numerically the existence of more than three steady states of (1) according to that positive parameter Q. The idea is to compute the evolution solution by a finite volume method (see [8]) by considering a sufficiently large time $T$. More precisely, we use a finite volume method with Weighted Essentially Non-Oscillatory (WENO) reconstruction in space and third-order Runge-Kutta TVD for time integration. For each time step, we compute a numerical solution of the EBM model equation for each cell of $\Gamma_{0}$, $u_{i}^{n+1}$, where $i$ represents the spatial control volume and $n+1$ is the time step. Then we use $u_{i}^{n+1}$ as a Dirichlet boundary condition for the DOM to obtain $U_{i, j}^{n+1}$. We prescribe the initial datum $\left(U_{0}, u_{0}\right)$ and the tolerance $\tau$ and compute the numerical solution for the corresponding large time $T$. This large time $T$ depends on the initial datum and the distance between two computed solutions for two consecutive times.

We collect a suitable number of initial datum $\left(U_{0}, u_{0}\right)$ and compute the $\omega$-limit set, $\omega\left(U_{0}, u_{0}\right)$ (see [9] and [10]). This allows us to obtain different limits, and so, different steady states. In particular, we have found a family of stationary solutions which is uniformly bounded.

\section{CONCLUSIONS}

We have improved the result concerning the number of stationary solutions for a coupled model (surface - deep ocean). It is known that for the values of the Solar parameter $Q$ belonging to the estimated interval $\left(Q_{2}, Q_{3}\right)$ the model has at least three stationary solutions. This was proved analytically in [7]. Now, we have found numerically more than three stationary solutions by using a finite volume method for the evolution model.

\section{ACKNOWLEDGMENTS}

This work is partially supported by the research projects MTM2017-85449-P and MTM2017-83391-P of Ministerio de Economía, Industria y Competitividad (Spain).

\section{REFERENCES}

[1] R.G. Watts and M. Morantine, Climatic Change 16, 83-97 (1990).

[2] P. Stone, J. Atmos. Sci. 29, 405-418 (1972).

[3] J.I. Diaz, A. Hidalgo and L. Tello, Proceedings of the Royal Society of London A: Mathematical, Physical and Engineering Sciences 470(2170):20140376 (2014).

[4] G. Hetzer and L. Tello, Dynamic Syst. Appl. 11, 381-402 (2002).

[5] M. I. Budyko, Tellus 21, 611-619 (1969).

[6] W. D. Sellers, Journal of Applied Meteorology 8, 392-400 (1969).

[7] J.I. Diaz and L. Tello, Discrete and Continuous Dynamical Systems - Series S (DCDS-S) 1, 253-262 (2008).

[8] A. Hidalgo and L. Tello, Discrete and Continuous Dynamical Systems 35 (4), 1503-1519 (2015).

[9] A. Hidalgo, L. Tello and E.F. Toro, Journal of Mathematical Biology 68, 1785-1814 (2014).

[10] A. Hidalgo and L. Tello, Computers \& Fluids 169, 380 - 387 (2018). 